Please do not remove this page

RMIT

UNIVERSITY

\title{
Oxygen-deficient strontium titanate based stretchable resistive memories
}

Rahman, Md. Ataur; Ahmed, Taimur; Walia, Sumeet; Sriram, Sharath; Bhaskaran, Madhu

https://researchrepository.rmit.edu.au/esploro/outputs/9921863221801341/filesAndLinks?institution=61 RMIT_INST\&index=null

Rahman, M. A., Ahmed, T., Walia, S., Sriram, S., \& Bhaskaran, M. (2018). Oxygen-deficient strontium titanate based stretchable resistive memories. Applied Materials Today, 13, 126-134.

https://doi.org/10.1016/j.apmt.2018.08.011

Document Version: Accepted Manuscript

Published Version: https://doi.org/10.1016/j.apmt.2018.08.011

Repository homepage: https://researchrepository.rmit.edu.au

(c) 2018 Elsevier Ltd. All rights reserved.

Downloaded On 2023/04/26 19:57:30 +1000

Please do not remove this page 
Thank you for downloading this document from the RMIT Research Repository.

The RMIT Research Repository is an open access database showcasing the research outputs of RMIT University researchers.

RMIT Research Repository: http://researchbank.rmit.edu.au/

\section{Citation:}

Rahman, M, Ahmed, T, Walia, S, Sriram, S and Bhaskaran, M 2018, 'Oxygen-deficient strontium titanate based stretchable resistive memories', Applied Materials Today, vol. 13, pp. 126-134.

See this record in the RMIT Research Repository at:

https://researchbank.rmit.edu.au/view/rmit:49576

Version: Accepted Manuscript

Copyright Statement:

(C) 2018 Elsevier Ltd. All rights reserved.

\section{Link to Published Version:}

https://doi.org/10.1016/j.apmt.2018.08.011 


\title{
Oxygen-deficient strontium titanate based stretchable resistive memories
}

Md. Ataur Rahman*, Taimur Ahmed, Sumeet Walia, Sharath Sriram and Madhu Bhaskaran*

Functional Materials and Microsystems Research Group and the Micro Nano Research Facility, RMIT University, Melbourne, VIC 3001, Australia

*Corresponding authors email: mdataur.rahman@student.rmit.edu.au madhu.bhaskaran@rmit.edu.au

\begin{abstract}
A stretchable non-volatile resistive memory is a fundamental element in realising complex neuromorphic computing and compact logic application adaptable to wearable electronics. A room temperature deposited $\mathrm{SrTiO}_{3-x}$ (STO) based resistive memory on stretchable polydimethylsiloxane (PDMS) substrate has been developed. The STO-based resistive memory does not require energy-intensive electroforming, exhibits stable complementary switching, long retention time, and reproducible endurance switching. The devices demonstrate the ability to operate under uniaxial tensile strain and extreme bending conditions. This work is an important step realising metal oxide based stretchable nonvolatile memories, critical to integrated devices for skin-mounted electronics.
\end{abstract}

Keywords: stretchablity, resistive memory, complementary switching, tensile strain, photoluminescence 


\section{Introduction}

A rapid increase in the demand for wearable and mechanically-conformable electronics in applications such as electronic skin, wearable sensors, human body monitoring and other biointegrated devices calls for every individual element of a device to be pliable. Wearable electronics therefore requires a degree of stretchability so that the device can withstand the stretch of the skin which is typically from (7-18)\% [1]. For a fully wearable integrated circuit, a degree of stretchability is desirable in its one of the most important circuit element: the memory unit.

In this context, polymer-based bistable resistive memory devices have been studied extensively [2]. This typically involves active layers that are coated using a variety of deposition techniques primarily sputtering [3-6], plasma enhanced atomic layer deposition [7] and plasma oxidized metal [8]. Another problem associated with these systems is the weak adhesion of the metal electrodes to the polymeric platforms when a stacked configuration is fabricated which can lead to the unreliable performance of the devices [9-11].

Although the demonstration of flexible memory systems is quite prevalent in literature [12, 13], reports on stretchable memory devices are limited only to the functional materials that are polymers such as a poly(butylthiophene)/poly(methyl methacrylate) blending film and poly[2,7-(9,9-dihexylfluorene)] [14, 15]. These organic nanostructures integrated with soft substrates have successfully replicated the performance of advanced CMOS technology, but at the cost of complicated and tedious fabrication processes, which are difficult to implement on large scale with existing manufacturing facilities $[16,17]$. Also, there is no comprehensive report to realize cross point and cross array structures of stretchable resistive memory using organic materials. Although organic materials seem to be a natural choice for stretchable memory, metal oxides are more amenable to doping based control and are chemically more stable [18]. Metal oxides, whose rich physics span the electrical [19], optical [20] and 
acoustic domain [21], offer a low-cost alternative for large-scale electronic memories. These materials can be deposited on large scale [22] with controllable electron mobilities [23], offer tunable band gap and high density integration due to their easier batch processing [16]. Several metal oxides can also be engineered in a low-dimensional form making them promising switching materials, although they remain in early stage of development in flexible and stretchable functional systems [24]. Metal oxide based devices compatible with batch processing are commonly deposited at high temperature and are naturally brittle (which render them not inherently stretchable). Most stretchable substrates such as poly dimethyl siloxane (PDMS) cannot withstand high temperature processing and also have significantly different mechanical properties to those of oxides. Due to this two-pronged problem, the most promising resistive switching materials such as binary and complex oxide systems are ruled out in stretchable platforms.

To address this bottleneck in the realisation of stretchable memory systems, we report a room temperature deposited $\mathrm{SrTiO}_{3-x}$ (STO) based resistive memory fabricated on stretchable PDMS platform as represented in Fig. 1. STO is selected as the functional oxide due to its ability to harbour oxygen vacancies $\left(V_{\mathrm{o}} \mathrm{s}\right)$ in the lattice and their concentration which can be controlled $[25,26]$. Also it has shown tremendous potential for future state-of-the-art memory systems [27-29]. A combination of ultraviolet (UV) and ultraviolet ozone (UVO) treatment is performed to ensure strong adhesion of the active oxide layer (STO) and metal electrodes to the PDMS surface (see Experimental) [30]. The fundamental memory characteristics of the $\mathrm{Au} / \mathrm{Ti} / \mathrm{STO} / \mathrm{Au} / \mathrm{Cr}$ stack are acquired under varying degrees of tensile strain. In addition, our stretchable devices are showing electroforming-free resistive switching characteristics which is typically required for energy-efficient memory systems [31]. Furthermore, a switching mechanism is proposed based on the electrical and compositional characterisation of the 
devices. Photoluminescence (PL) is also used as a non-destructive tool to map the effects of strain and further understand the switching characteristics of the devices.

\section{Experimental section}

\subsection{Material deposition and device fabrication}

Sylgard 184A (base) and Sylgard 184B (curer), the two parts manufactured by Dow-Corning, were mixed in the ratio of 10:1 (w/w) and stirred rigorously in a plastic cup using a plastic rod. The air bubbles trapped in the mixture were removed by applying gentle vacuum in the desiccator for $30 \mathrm{~min}$. The mixture was spin coated on a RCA-cleaned Pt-coated 3" Si wafer to obtain $250 \mu \mathrm{m}$ thick elastomers. Platinum of $20 \mathrm{~nm}$ thickness was used on Si to facilitate the peeling off of PDMS as the Pt has very poor adhesion with Si. Then, it was cured at room temperature for 72 hours in vacuum oven to form the cross-linked PDMS network and to remove any further trapped toluene. Before doing any photolithographic steps, PDMS/Pt/Si was subjected to $3 \mathrm{~min}$ of ultraviolet (UV) treatment due to which the macromolecules of PDMS surface undergo chain scission, including both the main chain backbone and side groups. Then, it was exposed to $30 \mathrm{~s}$ of ultraviolet ozone (UVO) to modify the near surface structure of Sylgard 184A. The molecular Oxygen and Ozone created during UVO treatment interacts with UV modified specimen to create a large number of hydrophilic $-\mathrm{OH}$ group as well as very thin layer of silica pillar on hydrophobic PDMS surface which facilitates photolithographic steps and lift off processes [30].

The room temperature deposited STO based resistive switching memories were fabricated by standard photolithography, thin film deposition and lift-off processes. Each cell comprises of $a$-STO sandwiched between $\mathrm{Cr} / \mathrm{Au}$ and $\mathrm{Ti} / \mathrm{Au}$ electrodes. After patterning through a photolithography (chrome) mask of 5214E image reversal photoresist on surface modified PDMS, the bottom electrodes consist of $\mathrm{Cr}(5 \mathrm{~nm})$ and $\mathrm{Au}(15 \mathrm{~nm})$ were deposited by 
electron beam evaporation (Kurt J. Lesker PVD75 Pro-line, which has the substrate cooling facilities) at a pressure of $1.5 \times 10^{-7}$ Torr, a deposition rate of $0.3 \AA$ As and room temperature. Functional bottom electrode was achieved after clean lift-off processes. $a$-STO of $30 \mathrm{~nm}$ thickness was deposited by RF sputtering (Kurt J. Lesker PVD75 sputtering system) in 100\% Argon atmosphere at a process pressure of $3.5 \times 10^{-7}$ Torr at room temperature from a commercial ceramic STO target (99.95\%, Testbourne Ltd) by using $200 \mathrm{~W}$ RF (13.54 MHz) plasma after a similar photolithographic process. Following the same photolithography process as earlier, top electrodes containing titanium $\mathrm{Ti}$ of $10 \mathrm{~nm}$ and $\mathrm{Au}$ of $60 \mathrm{~nm}$ thicknesses were deposited by electron beam evaporation. Choice of electrode materials was to ensure inert properties, to minimise their influence on oxygen vacancy transport in the oxide-based memory devices. Finally, the PDMS with fabricated devices was peeled off from the Si wafer.

\section{$2.2 \mathrm{X}$-ray photoelectron spectroscopy}

X-ray photoelectron spectroscopy (XPS) analysis was carried out by a Thermo Scientific KAlpha instrument equipped with an aluminium $K \alpha$ radiation source with energy of $1486.7 \mathrm{eV}$. The core-level elemental spectra were collected from a bare STO thin film deposited directly on PDMS substrate which was peeled off from Si. The surface of the $a$-STO on PDMS was in-situ cleaned by argon under high vacuum $\left(<5 \times 10^{-8}\right.$ Torr $)$ before collecting data using X-ray beam of $400 \mu \mathrm{m}$ in diameter. It was ensured that Ar assisted cleaning caused minimal oxygen removal when a short $(<5 \mathrm{~s})$ argon milling step with low beam energy $(<200 \mathrm{eV})$ was used. The binding energies of all principal elements were referenced corresponding to the adventitious carbon binding energy (C $1 s)$ of $285 \mathrm{eV}$. The standard Gaussian-Lorentzian function followed by the Shirley background correction was used to resolve all the spectra.

\subsection{Electrical characterization}


The current-voltage $(I-V)$ characteristics of these resistive random-access memory devices were carried out in an ambient atmosphere using an Agilent B2912A source meter semiconductor characterization system for two-probe measurements.

\subsection{Photoluminescence spectroscopy}

The photoluminescence (PL) emission spectra were obtained by using a Horiba Scientific FluoroMax-4 spectrofluorometer. All spectra were collected at room temperature, with $300 \mathrm{~nm}$ excitation wavelength; from the room temperature deposited bare STO thin film on PDMS. A custom-built scanning confocal fluorescence microscope with a high numerical aperture (0.9) objective was used for PL mapping. A $405 \mathrm{~nm}$ laser as an excitation source combined with avalanche photo-diode (APD, SPCM-AQRH-14) was employed for imaging and a spectrometer (Princeton instruments, SpectrPro) with a PIXISCCD camera to collect spectra. The lateral resolution was set to $300 \mathrm{~nm}$ with dwelling time of $0.5 \mathrm{~ms}$.

\section{Compositional analysis of STO}

Fig. 2 depicts the core-level XPS spectra of the major elements of our as-deposited STO thin film on PDMS. The core-level Sr $3 d$ spectra are fitted at binding energies of $132.98 \mathrm{eV}$ and $134.72 \mathrm{eV}$ for $\mathrm{Sr} 3 d 5 / 2$ and $\mathrm{Sr} 3 d 3 / 2$, respectively for the $\mathrm{Sr}^{2+}$ oxidation state [32](Fig. 2a). The Ti $2 p$ spectra are fitted with two distinct components at binding energies of $458.4 \mathrm{eV}$ and $456.2 \mathrm{eV}$ indexed to the $\mathrm{Ti}^{4+}$ and $\mathrm{Ti}^{(4-\delta)^{+}}$oxidation states, respectively [33](Fig. 2b). Furthermore, the core-level spectra of $\mathrm{O} 1 \mathrm{~s}$ are fitted by two components at $529.5 \mathrm{eV}$ and $531.4 \mathrm{eV}$ binding energies indexed to the $\mathrm{O}^{2-}$ oxidation state and $\mathrm{C}-\mathrm{O}$ bonds on the oxide surface, respectively, as shown in Fig. 2c. No prominent shift for the $\operatorname{Sr} 3 d_{3 / 2}$, Sr $3 d_{5 / 2}, \mathrm{Ti} 2 p$ or O $1 s$ core levels is observed [34]. It is well established that the presence of solely a $\mathrm{Ti}^{4+}$ component corresponds to a fully stoichiometric STO oxide, while the $\mathrm{Ti}^{(4-\delta)+}$ represents the 
presence of $V_{\mathrm{o}} \mathrm{s}$ [31] in our sputtered STO. Oxygen vacancies are generally introduced when the film is grown under an oxygen-deficient environment [33]. Under these growth conditions, according to the global charge equilibrium, one oxygen vacancy is created when two $\mathrm{Ti}^{4+}$ ions are transferred into two $\mathrm{Ti}^{3+}$ ions. Therefore, the precise chemical formula of the oxygen deficient STO thin film may be written as [35]:

$$
\mathrm{Sr}^{2+} \mathrm{Ti}_{1-2 x}^{4+} \mathrm{Ti}_{2 x}^{3+} \mathrm{O}_{3-x}^{2-}
$$

where $x$ corresponds to loss of oxygen from the stoichiometric compound. Moreover, the uniform oxygen deficiency distribution throughout our as-grown STO thin film is revealed from the depth profile as shown in Figure S1 which is in agreement with the earlier reported literature $[28,36,37]$. This oxygen deficient thin film plays vital role in forming oxygen vacancy induced conductive pathway which is discussed in detail in the following section.

\section{Results and discussions}

\subsection{Electroforming-free behaviour}

Fig. 3 shows the typical $I-V$ characteristics obtained using $\mathrm{Au} / \mathrm{Ti} / \mathrm{STO} / \mathrm{Au} / \mathrm{Cr}$ metalinsulator-metal (MIM) structures on PDMS. It is observed from the $I-V$ characteristics that when the applied field across the memory device is in the range of $-0.2 \mathrm{~V}$ to $+0.2 \mathrm{~V}$, the maximum resistance across the oxide is $4.7 \mathrm{k} \Omega$. This indicates that at such low applied voltages, the density of field induced oxygen vacancies is insignificant inside the films [38]. Another feature that should be noted is that, unlike most transition metal oxides that require an initial electroforming sweep to activate their resistive switching behaviour, our devices are electroforming-free in nature as depicted in Figure S2.

We postulate two possible reasons for these devices being 'electroforming-free' in nature. Firstly, the impact of the mechanical properties of the underlying metal layer of a STO thin 
film play a significant role in the migration of oxygen vacancies when an electric field is applied. Al-Hamadany et al. [29] have modelled that compressive and tensile bi-axial strains can result in the migration of oxygen vacancies $\left(V_{\mathrm{o}} \mathrm{s}\right)$ in STO films. By using spin-polarized density functional theory (DFT), they proposed that compressive strain is a potential route for limiting injection of electrically active $V_{o}$ centres, whereas tensile strain enhances diffusion of oxygen vacancies [39]. Bi-axial tensile strain of Platinum $(\mathrm{Pt})$ is $2.2 \%$ [40], half of the strain of Gold $(\mathrm{Au}) 4.4 \%$ [41], creates less strain on the as-deposited STO. This will potentially result in the migration of a larger number of $V_{o}$ s which govern the electrical properties of STO in a stacked MIM configuration in case of Au based devices. This is further confirmed by electrical outcomes as shown in Figure $\mathrm{S} 2$ which depicts that the devices with the $\mathrm{Pt}$ underlying layer of STO thin film required energy intensive electroforming voltage, whereas, the devices with Au bottom layer showed electroforming free behaviour. Additionally, it is also expected that the oxygen vacancies will align along the direction of strain in the films, which can inadvertently cause the formation of random conductive pathways in the oxide films.

Secondly, the electrical conductivity of $\mathrm{SrTiO}_{3-x}$ increases as $x$ increases. In our study, we sputtered STO in Ar environment (see Experimental for details) which results in the deposition of oxygen-deficient STO as also highlighted by the XPS analysis (Fig. 2). The inherent $V_{\mathrm{o}} \mathrm{s}$ in the $\mathrm{Ti}-\mathrm{O}_{6}$ octahedra that are created by preferentially removing the oxygen atoms due to heavy $\mathrm{Ar}^{+}$ion bombardment during the sputtering process, generates conduction band electrons in the $\mathrm{Ti} 3 d$ states [42]. Moreover, due to the oxygen deficiencies, the $\mathrm{TiO}_{2}$ in STO is more inclined to a semi-metallic state $\left(\mathrm{TiO}_{2-x}\right)$ rather than the insulating $\mathrm{TiO}_{2}$. As such, the semi-metallic $\mathrm{Ti}$ in our $\mathrm{SrTiO}_{3-x}$ films may be responsible for the forming-free process partly because of its high content in the film. Furthermore, when the $\mathrm{Au} / \mathrm{Cr}$ bottom electrode is biased, the initially oxidised $\mathrm{Sr}$ and $\mathrm{Ti}$ species (as revealed from the XPS 
analysis) in the as-deposited state may be reduced by the applied electric field which leads to an increase in electrical conductivity. So, it is reasonable to ascribe the forming free behaviour in our STO films to the specific deposition conditions and the internal microstructural changes induced due to the underlying higher tensile strain electrode. When we apply higher bias sweeps of $-1 \mathrm{~V}$ to $1 \mathrm{~V}$, the resistance decreases nonlinearly with a higher slope. As the range of the applied bias is extended further to cover $-1.6 \mathrm{~V}$ to $+1.6 \mathrm{~V}$, threshold switching behaviour emerges with a sudden increase in current observed at $0.75 \mathrm{~V}$ [43].

Fig. 4 illustrates the possible resistive switching mechanism based on the electrical and material characterization of our complementary switching devices. In the virgin state, the reduced metallic ions and $V_{\mathrm{o}} \mathrm{s}$ generated due to the $\mathrm{Au}$ induced strain effects are randomly distributed which can be aligned with applying a very small negative voltage to the bottom electrode consequently offering electroforming free characteristics as depicted in Fig. 4a. When the negative voltage is increased, a threshold voltage switches the devices to an $\mathrm{ON}_{1}$ state where all semi-metallic ions and oxygen vacancies are aligned between the top and bottom electrode with a higher concentration towards the bottom electrode as illustrated in Fig. 4b. A larger magnitude of the negative voltage results in a higher concentration of oxygen vacancies towards the bottom electrode, which creates an inadequacy of oxygen vacancies around the top electrode as shown in Fig. 4c, switching the device to a $S^{0}$ state. Subsequently, an application of positive voltage at the bottom electrode repels the positively charged oxygen vacancies towards the top electrode which results in an intermediate $\mathrm{ON}_{2}$ state before switching to the $\mathrm{S}^{1}$ state as the magnitude is increased (Fig. 4d, e).

\subsection{Complementary switching behaviour}


Fig. 5a depicts the symmetric complementary switching CS behaviour seen in our devices for 100 continuous switching cycles. The combination of the presence of inherent oxygen vacancies along with randomly oriented pathways that are likely created due to the underlying electrode induced strain result in the migration of $V_{\mathrm{o}} \mathrm{s}$ under an applied bias. The observed CS behaviour occurs due to the alternate growth and rupture of the depletion region in the vicinity of one of the top/bottom interfaces [44] depending on the polarity of the applied bias. The diminishing of depletion region results in a local low resistivity at one of the interfaces (LRS), whereas enhancement of the depletion region causes local high resistivity (HRS) near the interface. When a negative bias is applied at the bottom Au/STO interface, the device switches to an $\mathrm{ON}_{1}$ state, where the conductive pathway is continuous with no depletion interface. On further increasing the magnitude of the applied bias, the $S^{\mathbf{0}}$ state is achieved. In the $S^{\mathbf{0}}$ state, the $V_{\mathrm{o}}$ s are concentrated towards the bottom electrode (LRS) there by creating a partial depletion region at the top Ti/STO interface (HRS). Conversely, when a positive voltage is applied to the bottom electrode, the $V_{\mathrm{o}} \mathrm{s}$ are concentrated at the top Ti/STO interface (LRS) resulting in a sequential transition from the $\mathrm{ON}_{2}$ transition state to the $S^{1}$ state where a depletion region is enhanced in the vicinity of the bottom Au/STO interface (HRS). The corresponding switching cycles in linear scale are depicted in Figure S3.

In contrast to the bipolar ReRAM devices, the CS device only offers $I-V$ based readout to retrieve the stored information from the CS cell at high voltage, since the two high resistive storing states are not distinguishable by a non-destructive readout at low voltage [44]. As a consequence, the device retention and endurance tests are performed at a read voltage of $1.0 \mathrm{~V}$ so that the device is in the $\mathrm{ON}$ state $\left(\mathrm{ON}_{1}\right.$ or $\left.\mathrm{ON}_{2}\right)$. Fig. $5 \mathrm{~b}$ depicts the stable retention characteristics for $10^{3} \mathrm{~s}$ with an average switching ratio of $\sim 6$. The presence of distinguishable states $S^{\mathbf{0}}$ and $S^{\mathbf{1}}$ indicate the stability of the CS behaviour in our MIM device. 
As the real input signal to a practical integrated circuit is closer to being a pulse, therefore endurance measurement in this work is conducted in pulse mode operation with duration of $50 \mu \mathrm{s}$ as shown in Fig. 5c. For each complete cycle, a negative pulse with an amplitude of 1.6 $\mathrm{V}$ switches the devices to a $\mathrm{S}^{\mathbf{0}}$ state followed by a positive read voltage of $1.0 \mathrm{~V}$. This sequence is followed by a positive pulse with an amplitude of $1.6 \mathrm{~V}$ which switches the device to the $S^{1}$ state and a read voltage of positive $1.0 \mathrm{~V}$. The read voltage after the negative and positive pulses, switches the devices from low resistance to high resistance state respectively.

The endurance characteristics are evaluated for 2000 cycles indicating stable CS behaviour. The slight variation of switching ratios after 1500 cycles can be attributed to the cyclinginduced degradation due to relatively higher $V_{\text {READ }}$ of $1 \mathrm{~V}$ as shown in Fig. $5 \mathrm{~d}$.

\subsection{Complementary switching behaviour under tensile strain}

To observe the effect of strain on the switching characteristics of our MIM devices, we applied a sequence of varying strain (0-6\%) using a custom-built fully-automated stretching stage with a step resolution of $2.5 \mu \mathrm{m}$ as shown in Fig. 6a. The displacement of the samples was controlled by a linear stage actuated with a micrometre screw gauge. The measurements at every strain level are acquired for 5 cycles. As the applied strain is gradually increased from $0-4 \%$, the switching voltage of the CS device to initiate the $S^{0}$ and $S^{1}$ states increase from an amplitude of $1.6 \mathrm{~V}$ at $0 \%$ to $2.4 \mathrm{~V}$ at $4 \%$ as shown in Fig. $6 \mathrm{c}$ whereas Figure S4 the curves in linear scale. The electrical retention stability and cycling endurance under tensile strain from $0-4 \%$ are depicted in Fig. 6d,e for $100 \mathrm{~s}$ and 100 cycles, respectively. With an increasing strain, the switching voltage and read voltage increased, while the switching ratio decreased from $\sim 6.0$ at $0 \%$ to $\sim 4.5$ under $4 \%$ strain. No electrical conductivity is seen at $6 \%$ strain indicating device reaches an open-circuit state. As the uniaxial tensile strain increases, 
the inter layer lattice planes separation decreases. The in-plane and inter-plane Ti ions that are separated by a single $\mathrm{SrO}$ plane get closer [27]. Upon migration of any oxygen ions from the $\mathrm{SrO}$ plane due to the tensile strain, the nearest-neighbour Ti-ions experience an electrostatic repulsion and move apart. Hence, there is a general and substantial increase in barrier heights for all charge states with increasing strain. Oxygen vacancy diffusion along this larger barrier is rendered unfavourable under strain [39]. As the barrier height increases with tensile strain, it results in an increase in the switching voltages required to turn the devices to the $\mathrm{ON}$ state (i.e., $\mathrm{ON}_{1}$ or $\left.\mathrm{ON}_{2}\right)$. A further increase in strain $(>6 \%)$, results in device ceasing switching most likely due to the micro-crackings in the top and bottom electrodes as well as the functional STO layer. Such micro-cracking is a known phenomenon in well-adhered metal thin films on elastomeric substrates stretched beyond their elastic limit [45]. The micro-cracking allows the thin film to deform beyond their bulk rupture strain and returns to its initial position [11], which is critical for long-term cyclic use of such devices. To further validate, we investigate the morphology of electrodes and active layer using Scanning Electron Microscope (SEM) under varying degree of strain. We found that the strain beyond $4 \%$ shows clear evidence of micro-cracks on the active layer as well as on the electrodes as depicted in Figure S5. Interestingly, upon removing the strain after $8 \%$, the micro cracks almost disappeared which explain the stability of the device under multiple stretching-releasing cycling. Fig. $6 f$ illustrates the successive recovery of the device to its initial position ( $0 \%$ strain) after being subjected to tensile strains up to $4 \%$. This indicates the stability of the memristive behaviour under strain. A stable and repeatable switching behaviour is observed during the stretching and releasing cycles (Figure S6). Moreover, to test the memory behaviour after 100 stretch-release cycles, micrometer screw of the stretching stage was driven by a stepper motor (Trinamic PD-110-42, Germany). The stepper motor was controlled by a LabView program which applies displacement mapping 
automatically. The linear switching behaviour for 50 cycles after 100 stretch-release cycles at 4\% strain is represented in Figure S7 indicating stable stretchable behaviour of our devices. Furthermore, the switching characteristics of our devices are also acquired under flex by affixing samples to a cylinder of $7 \mathrm{~mm}$ diameter as shown in Fig. 6b. The results show stable switching in log scale as illustrated in Fig. $6 \mathrm{~g}$ and indicate that our devices also perform well on a curvilinear surface making it viable for being conformably contacted on any part of human body. Figure S8 illustrated the switching in linear scale.

\subsection{Photoluminescence measurements}

In order to gain insights into the effect of applied strain on the electronic structure of STO, we performed photoluminescence measurements on our sputtered STO thin films under varying degrees of strain $(0-8 \%)$. The PL phenomenon can be used to understand the electronic states within the band gap due to oxygen vacancies, defects, or impurities [46]. According to the literature, PL in STO is due to the recombination of electrons and holes, generated by distorted clusters containing oxygen vacancies, trapped in the intermediate states to the band gap [47]. As the PL response can be modulated by changing the density of oxygen vacancies [31], we demonstrate herein the effect of tensile strain on the PL emission using a $405 \mathrm{~nm}$ excitation source. According to the PL results shown in Fig. 7, the increase in tensile strain causes a reversible decrease in the intensity of PL emission. As the excitation energy does not change, the PL emission in STO can be related to the strain induced density of charge carriers (oxygen vacancies) in the samples. The band gap energy does not change significantly under applied tensile strain which is indicated by the position of the PL peak being unchanged [48]. As such, it can be inferred that an applied strain creates an

unfavourable barrier for the generated oxygen vacancies as discussed earlier. So, the PL spectra decreases with the increasing strain which results in increasing threshold voltages for the devices to switch to the ON state. Eventually, we can summarize from the electrical 
results and photoluminescence spectra that the threshold voltage increases due the reduction of $V_{\mathrm{o}} \mathrm{s}$ generation under lateral strain which potentially create an unfavourable condition for oxygen vacancy movement.

\section{Conclusion}

In conclusion, we have demonstrated forming-free complementary switching behaviour of room-temperature-deposited STO thin film fabricated on a stretchable PDMS substrate. It is confirmed that oxygen-deficient STO thin film along with underlying Au-induced oxygen vacancies play a great role on the forming-free nature of the devices. The resistive memories have exhibited stable non-volatile characteristics with an ON/OFF ratio of $\sim 6$, data retention time over $10^{3} \mathrm{~s}$ and cycling endurance of $2 \times 10^{3}$ cycles. The device showed reliable switching characteristics up to $4 \%$ lateral strain which may possibly be extended to at least $10 \%$ by modifying the geometry and size of electrodes, self-similar, or fractal electrode designs, downsizing the devices to sub nanometre level and reducing the thickness of substrate, electrode and active layers. The results have also elucidated that room temperature deposition is a viable pathway for overcoming the bottleneck of realising metal oxide based stretchable resistive memories. Thus, the experimental results highlight the feasibility of metal oxide based stretchable resistive memory and could pave the way for fully-integrated, skin-mounted electronics.

\section{Acknowledgements}

The authors acknowledge support from the Australian Research Council (ARC) for personnel and project support via DE160100023 (M.B.) and DP130100062 (S.S.). The authors would like to acknowledge the technical assistance of the Micro Nano Research Facility (MNRF) and the RMIT Microscopy and Microanalysis Research Facility (RMMF). Furthermore, the authors extend acknowledgment to Dr. Anthony Hope and Dr. Desmond W.M. Lau of ARC 
Centre of Excellence for Nanoscale Bio-Photonics \& School of Science, RMIT University, for their technical support to avail Horiba Scientific FluoroMax-4 spectrofluorometer.

\section{Appendix A: Supplementary information}

Supplementary data associated with this article can be found, in the online version. 


\section{$\underline{\text { References }}$}

[1] P. Gutruf, E. Zeller, S. Walia, H. Nili, S. Sriram, M. Bhaskaran, Stretchable and Tunable Microtectonic ZnO-Based Sensors and Photonics, Small, 11 (2015) 4532-4539.

[2] B.C. Jang, H. Seong, S.K. Kim, J.Y. Kim, B.J. Koo, J. Choi, S.Y. Yang, S.G. Im, S.Y. Choi, Flexible Nonvolatile Polymer Memory Array on Plastic Substrate via Initiated Chemical Vapor Deposition, ACS Appl Mater Inter, 8 (2016) 12951-12958.

[3] J. Won Seo, J.-W. Park, K.S. Lim, S.J. Kang, Y.H. Hong, J.H. Yang, L. Fang, G.Y. Sung, H.-K. Kim, Transparent flexible resistive random access memory fabricated at room temperature, Appl Phys Lett, 95 (2009) 133508.

[4] C.H. Cheng, F.S. Yeh, A. Chin, Low-power high-performance non-volatile memory on a flexible substrate with excellent endurance, Adv Mater, 23 (2011) 902-905.

[5] H. Zhou, G.-J. Fang, Y. Zhu, N. Liu, M. Li, X.-Z. Zhao, Flexible resistive switching memory based on $\mathrm{Mn}_{0.20} \mathrm{Zn}_{0.80} \mathrm{O} / \mathrm{HfO}_{2}$ bilayer structure, J Phys D, 44 (2011) 445101.

[6] S. Mondal, C.-H. Chueh, T.-M. Pan, High-Performance Flexible Ni/Sm ${ }_{2} \mathrm{O}_{3} / \mathrm{ITO}$ ReRAM Device for Low-Power Nonvolatile Memory Applications, IEEE Electr Device L, 34 (2013) 1145-1147.

[7] S. Kim, O. Yarimaga, S.-J. Choi, Y.-K. Choi, Highly durable and flexible memory based on resistance switching, Solid-State Electron, 54 (2010) 392-396.

[8] S. Kim, Y.-K. Choi, Resistive switching of aluminum oxide for flexible memory, Appl Phys Lett, 92 (2008) 223508.

[9] L. Liang, K. Li, C. Xiao, S. Fan, J. Liu, W. Zhang, W. Xu, W. Tong, J. Liao, Y. Zhou, B. Ye, Y. Xie, Vacancy associates-rich ultrathin nanosheets for high performance and flexible nonvolatile memory device, J Am Chem Soc, 137 (2015) 3102-3108.

[10] K. Qian, R.Y. Tay, V.C. Nguyen, J. Wang, G. Cai, T. Chen, E.H.T. Teo, P.S. Lee, Hexagonal Boron Nitride Thin Film for Flexible Resistive Memory Applications, Adv Funct Mater, 26 (2016) 2176-2184.

[11] P. Gutruf, S. Walia, M. Nur Ali, S. Sriram, M. Bhaskaran, Strain response of stretchable micro-electrodes: Controlling sensitivity with serpentine designs and encapsulation, Appl Phys Lett, 104 (2014) 021908.

[12] S.K. Hwang, J.M. Lee, S. Kim, J.S. Park, H.I. Park, C.W. Ahn, K.J. Lee, T. Lee, S.O.

Kim, Flexible multilevel resistive memory with controlled charge trap B- and N-doped carbon nanotubes, Nano Lett, 12 (2012) 2217-2221.

[13] M. Ghoneim, M. Hussain, Review on Physically Flexible Nonvolatile Memory for Internet of Everything Electronics, Electronics, 4 (2015) 424-479.

[14] Y.-C. Lai, Y.-C. Huang, T.-Y. Lin, Y.-X. Wang, C.-Y. Chang, Y. Li, T.-Y. Lin, B.-W. Ye, Y.-P. Hsieh, W.-F. Su, Y.-J. Yang, Y.-F. Chen, Stretchable organic memory: toward learnable and digitized stretchable electronic applications, NPG Asia Mater, 6 (2014) e87. [15] J.-T. Wang, K. Saito, H.-C. Wu, H.-S. Sun, C.-C. Hung, Y. Chen, T. Isono, T. Kakuchi, T. Satoh, W.-C. Chen, High-performance stretchable resistive memories using donoracceptor block copolymers with fluorene rods and pendent isoindigo coils, NPG Asia Mater, 8 (2016) e298.

[16] Y. Liu, M. Pharr, G.A. Salvatore, Lab-on-Skin: A Review of Flexible and Stretchable Electronics for Wearable Health Monitoring, ACS Nano, 11 (2017) 9614-9635.

[17] R. Ramanathan, A. Pearson, S. Walia, A.E. Kandjani, M. Mohammadtaheri, M. Bhaskaran, S. Sriram, S.K. Bhargava, V. Bansal, Solution-processable do-it-yourself switching devices (DIY devices) based on CuTCNQ metal-organic semiconductors, Applied Materials Today, 10 (2018) 12-17. 
[18] S. Walia, S. Balendhran, H. Nili, S. Zhuiykov, G. Rosengarten, Q.H. Wang, M. Bhaskaran, S. Sriram, M.S. Strano, K. Kalantar-zadeh, Transition metal oxides Thermoelectric properties, Progress in Materials Science, 58 (2013) 1443-1489.

[19] E.M.C. Fortunato, P.M.C. Barquinha, A.C.M.B.G. Pimentel, A.M.F. Gonçalves, A.J.S. Marques, R.F.P. Martins, L.M.N. Pereira, Wide-bandgap high-mobility ZnO thin-film transistors produced at room temperature, Applied Physics Letters, 85 (2004) 2541-2543.

[20] J. Zhou, Y. Gu, Y. Hu, W. Mai, P.H. Yeh, G. Bao, A.K. Sood, D.L. Polla, Z.L. Wang, Gigantic enhancement in response and reset time of ZnO UV nanosensor by utilizing Schottky contact and surface functionalization, Appl Phys Lett, 94 (2009) 191103.

[21] G. Zhu, R. Yang, S. Wang, Z.L. Wang, Flexible high-output nanogenerator based on lateral ZnO nanowire array, Nano Lett, 10 (2010) 3151-3155.

[22] K.K. Banger, Y. Yamashita, K. Mori, R.L. Peterson, T. Leedham, J. Rickard, H.

Sirringhaus, Low-temperature, high-performance solution-processed metal oxide thin-film transistors formed by a 'sol-gel on chip' process, Nat Mater, 10 (2011) 45-50.

[23] S.-J. Seo, C.G. Choi, Y.H. Hwang, B.-S. Bae, High performance solution-processed amorphous zinc tin oxide thin film transistor, Journal of Physics D: Applied Physics, 42 (2009).

[24] K. Kalantar-zadeh, J.Z. Ou, T. Daeneke, A. Mitchell, T. Sasaki, M.S. Fuhrer, Two dimensional and layered transition metal oxides, Applied Materials Today, 5 (2016) 73-89.

[25] A. Koehl, H. Wasmund, A. Herpers, P. Guttmann, S. Werner, K. Henzler, H. Du, J. Mayer, R. Waser, R. Dittmann, Evidence for multifilamentary valence changes in resistive switching $\mathrm{SrTiO}_{3}$ devices detected by transmission X-ray microscopy, APL Materials, 1 (2013) 042102.

[26] R. Muenstermann, T. Menke, R. Dittmann, R. Waser, Coexistence of filamentary and homogeneous resistive switching in Fe-doped $\mathrm{SrTiO}_{3}$ thin-film memristive devices, Adv Mater, 22 (2010) 4819-4822.

[27] M. Kubicek, R. Schmitt, F. Messerschmitt, J.L.M. Rupp, Uncovering Two Competing Switching Mechanisms for Epitaxial and Ultrathin Strontium Titanate-Based Resistive Switching Bits, ACS Nano, 9 (2015) 10737-10748.

[28] H. Nili, S. Walia, S. Balendhran, D.B. Strukov, M. Bhaskaran, S. Sriram, Nanoscale Resistive Switching in Amorphous Perovskite Oxide (a-SrTiO 3 ) Memristors, Adv Funct Mater, 24 (2014) 6741-6750.

[29] T. Ahmed, S. Walia, E.L.H. Mayes, R. Ramanathan, P. Guagliardo, V. Bansal, M. Bhaskaran, J.J. Yang, S. Sriram, Inducing tunable switching behavior in a single memristor, Applied Materials Today, 11 (2018) 280-290.

[30] K. Efimenko, W.E. Wallace, J. Genzer, Surface Modification of Sylgard-184

Poly(dimethyl siloxane) Networks by Ultraviolet and Ultraviolet/Ozone Treatment, J Colloid Interf Sci, 254 (2002) 306-315.

[31] T. Ahmed, S. Walia, J. Kim, H. Nili, R. Ramanathan, E.L.H. Mayes, D.W.M. Lau, O. Kavehei, V. Bansal, M. Bhaskaran, S. Sriram, Transparent amorphous strontium titanate resistive memories with transient photo-response, Nanoscale, 9 (2017) 14690-14702.

[32] P. Pal, P. Kumar, A. V, A. Dogra, A.G. Joshi, Chemical potential shift and gap-state formation in $\mathrm{SrTiO}_{3-\delta}$ revealed by photoemission spectroscopy, J Appl Phys, 116 (2014) 053704.

[33] H.L. Cai, X.S. Wu, J. Gao, Effect of oxygen content on structural and transport properties in $\mathrm{SrTiO}_{3-\mathrm{x}}$ thin films, Chem Phys Lett, 467 (2009) 313-317.

[34] S. Balaz, Z. Zeng, L.J. Brillson, Heterojunction band offsets and dipole formation at $\mathrm{BaTiO}_{3} / \mathrm{SrTiO}_{3}$ interfaces, J Appl Phys, 114 (2013) 183701. 
[35] R. Perez-Casero, J. Perrière, A. Gutierrez-Llorente, D. Defourneau, E. Millon, W. Seiler, L. Soriano, Thin films of oxygen-deficient perovskite phases by pulsed-laser ablation of strontium titanate, Phys Rev B, 75 (2007).

[36] K. Szot, W. Speier, G. Bihlmayer, R. Waser, Switching the electrical resistance of individual dislocations in single-crystalline $\mathrm{SrTiO}_{3}$, Nat Mater, 5 (2006) 312.

[37] S.S.A. Seo, Z. Marton, W.S. Choi, G.W.J. Hassink, D.H.A. Blank, H.Y. Hwang, T.W. Noh, T. Egami, H.N. Lee, Multiple conducting carriers generated in $\mathrm{LaAlO}_{3} / \mathrm{SrTiO}_{3}$ heterostructures, Appl Phys Lett, 95 (2009) 082107.

[38] X. Cao, X. Li, X. Gao, W. Yu, X. Liu, Y. Zhang, L. Chen, X. Cheng, Forming-free colossal resistive switching effect in rare-earth-oxide $\mathrm{Gd}_{2} \mathrm{O}_{3}$ films for memristor applications, J Appl Phys, 106 (2009) 073723.

[39] R. Al-Hamadany, J.P. Goss, P.R. Briddon, S.A. Mojarad, A.G. O'Neill, M.J. Rayson, Impact of tensile strain on the oxygen vacancy migration in $\mathrm{SrTiO}_{3}$ : Density functional theory calculations, J Appl Phys, 113 (2013) 224108.

[40] M. Mrovec, J.M. Albina, B. Meyer, C. Elsässer, Schottky barriers at transitionmetal/SrTiO ${ }_{3}(001)$ interfaces, Phys Rev B, 79 (2009).

[41] K.L. Zhao, D. Chen, D.X. Li, First principles study of interface structure and electronic property of $\mathrm{Au} / \mathrm{SrTiO}_{3}(001)$, Comp Mater Sci, 50 (2010) 98-104.

[42] H. Nili, S. Walia, A.E. Kandjani, R. Ramanathan, P. Gutruf, T. Ahmed, S. Balendhran, V. Bansal, D.B. Strukov, O. Kavehei, M. Bhaskaran, S. Sriram, Donor-Induced Performance Tuning of Amorphous $\mathrm{SrTiO}_{3}$ Memristive Nanodevices: Multistate Resistive Switching and Mechanical Tunability, Adv Funct Mater, 25 (2015) 3172-3182.

[43] E. Linn, R. Rosezin, C. Kugeler, R. Waser, Complementary resistive switches for passive nanocrossbar memories, Nat Mater, 9 (2010) 403-406.

[44] T. Breuer, A. Siemon, E. Linn, S. Menzel, R. Waser, V. Rana, A HfO ${ }_{2}$-Based Complementary Switching Crossbar Adder, Adv Electron Mater, 1 (2015) 1500138.

[45] S.P. Lacour, D. Chan, S. Wagner, T. Li, Z. Suo, Mechanisms of reversible stretchability of thin metal films on elastomeric substrates, Appl Phys Lett, 88 (2006) 204103.

[46] L. Gracia, J. Andrés, V.M. Longo, J.A. Varela, E. Longo, A theoretical study on the photoluminescence of $\mathrm{SrTiO}_{3}$, Chem Phys Lett, 493 (2010) 141-146.

[47] M.L. Crespillo, J.T. Graham, F. Agulló-López, Y. Zhang, W.J. Weber, Correlation between $\mathrm{Cr}^{3+}$ Luminescence and Oxygen Vacancy Disorder in Strontium Titanate under MeV Ion Irradiation, J. Phys. Chem. C, 121 (2017) 19758-19766.

[48] A.E. Souza, G.T.A. Santos, B.C. Barra, W.D. Macedo, S.R. Teixeira, C.M. Santos, A.M.O.R. Senos, L. Amaral, E. Longo, Photoluminescence of SrTiO3: Influence of Particle Size and Morphology, Crystal Growth \& Design, 12 (2012) 5671-5679. 


\section{Figure captions:}

Fig. 1 Physical structure of stretchable memory devices. (a) Schematic of a device with stack of $\mathrm{Au} / \mathrm{Ti} / \mathrm{STO} / \mathrm{Au} / \mathrm{Cr} / \mathrm{PDMS}$; where $\mathrm{Au} / \mathrm{Ti}$ works as top electrode (TE) and $\mathrm{Au} / \mathrm{Cr}$ as bottom electrode (BE). (b) Shows thickness of each layer on the cross section as mentioned in (a). (c) An optical micrograph of a fabricated cross-point device where the dotted square represents the oxide region of area $60 \times 60 \mu \mathrm{m}^{2}$. (d) An array of cross-point devices after peeling off from the Si carrier substrate. The standalone devices on $250 \mu \mathrm{m}$ thick PDMS are flexible as well as stretchable.

Fig. 2 X-ray photoelectron spectroscopic characterization of room temperature sputtered STO on PDMS. (a) The core level spectra of $\mathrm{Sr} 3 d$ showing $\mathrm{Sr}^{2+}$ oxidation state, (b) $\mathrm{Ti} 2 p$ with oxidation states $\mathrm{Ti}^{4+}$ and $\mathrm{Ti}^{(4-\delta)+}$, and (c) $\mathrm{O} 1 s$ representing $\mathrm{O}^{2-}$ oxidation state.

Fig. 3 Typical $I-V$ switching curve with the application of voltage sweeps. The bias was applied to the bottom electrode $\mathrm{Au} / \mathrm{Cr}$ and the top electrode $\mathrm{Au} / \mathrm{Ti}$ was grounded. The labels indicate the voltage sweeps used, starting at $0 \mathrm{~V}$, cycling through the negative and then positive voltages.

Fig. 4 Schematic illustration of possible complementary switching mechanism. (a) Randomly distributed oxygen vacancies and semi-metallic ions during the virgin state of the device. White, unfilled spheres represent the oxygen vacancies and red, filled spheres illustrate the metallic ions. The device goes to the ON state under negative bias as shown in (b) and positive half cycle as shown in (d). (c) Local low resistive state (LRS) at the bottom electrode and local high resistive state (HRS) at the top electrode create the $S^{0}$ state due to the negative bias at bottom electrode. (e) Local LRS at the top electrode and HRS at the bottom electrode constitute the $S^{1}$ state due to the positive bias applied the bottom electrode. While the top half 
circular arrow marks show the sequence of applied electric field, the bottom black arrow marks represent the strength of higher electric field.

Fig. 5 Resistive random-access memory performances. (a) Quasi-static sweep for 100 cycles in log scale. The arrow marks show the switching direction. The on state of negative half cycle is termed as $\mathrm{ON}_{1}$ and positive half cycle as $\mathrm{ON}_{2}$. (b) Retention for $1000 \mathrm{~s}$ at read voltage of $1 \mathrm{~V}$. The red and black circles represent the ON and OFF retention, respectively. (c) Pulse mode operation for 2 consecutive cycles. The applied $V_{-}-V_{\mathrm{READ}}-V_{+}-V_{\mathrm{READ}}$ voltage sequence cycles the device through the $\mathrm{ON}_{1}-\mathrm{S}^{0}-\mathrm{ON}_{2}-\mathrm{S}^{1}$ sequence. (d) Endurance for 2000 cycles at read voltage of $1 \mathrm{~V}$.

Fig. 6 (a) Stretching stage where the sample is attached between the sample holders to apply strain. (b) Curvilinear stage to test the device functionality under curvature. (c) Switching under tensile strain from $0 \%$ to $4 \%$ (inset: Linear scale). (d) Electrical retention stability under tensile strain from $0 \%$ to $4 \%$. (e) Electrical endurance stability under tensile strain from $0 \%$ to $4 \%$. (f) Effect of tensile strain on the switching voltage. (g) Switching on curvilinear surface with a radius of $3.5 \mathrm{~mm}$ in $\log$ scale.

Fig. 7 Effect of tensile strain on STO deposited on PDMS using photoluminescence spectra. (a) Uniaxial stretching from $0 \%$ to $8 \%$. PDMS substrate intensity remains same for the entire wavelength. (b) Removal of strain from $8 \%$ to $0 \%$. 


\section{FIGURES}

a

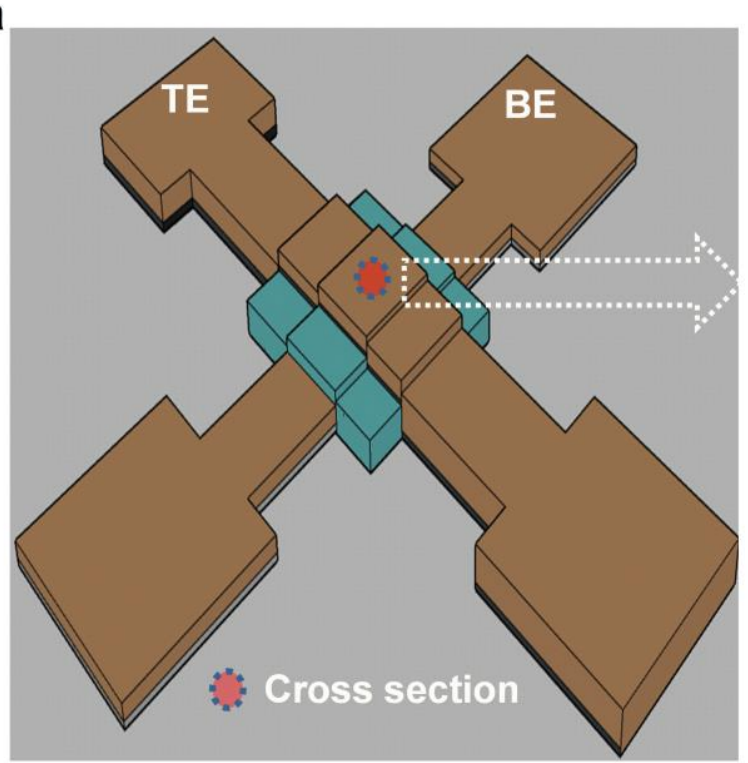

c

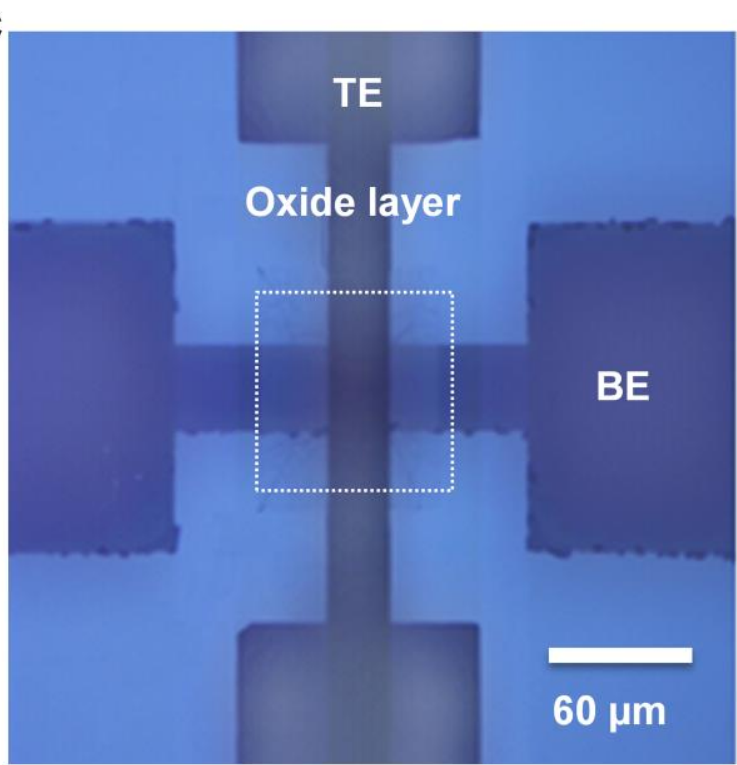

b

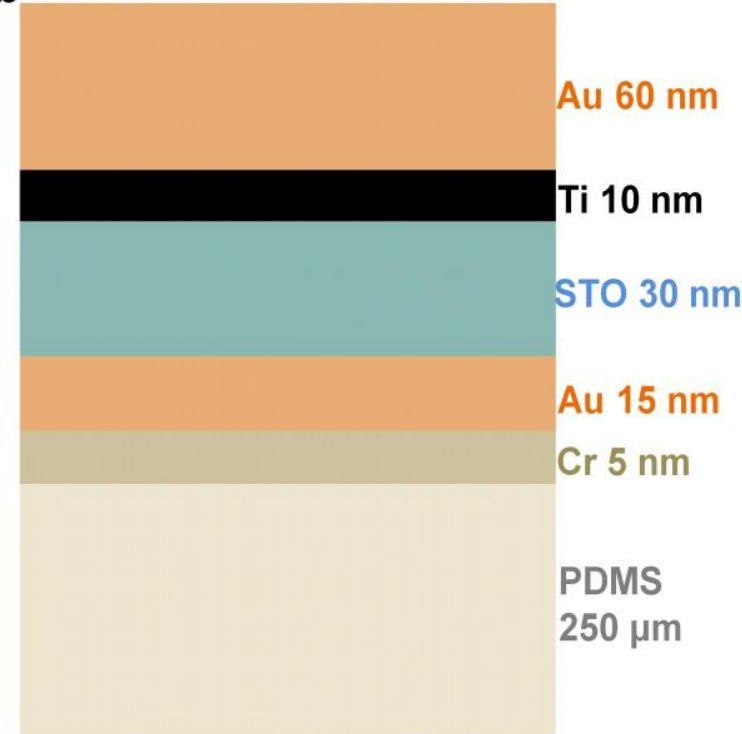

d

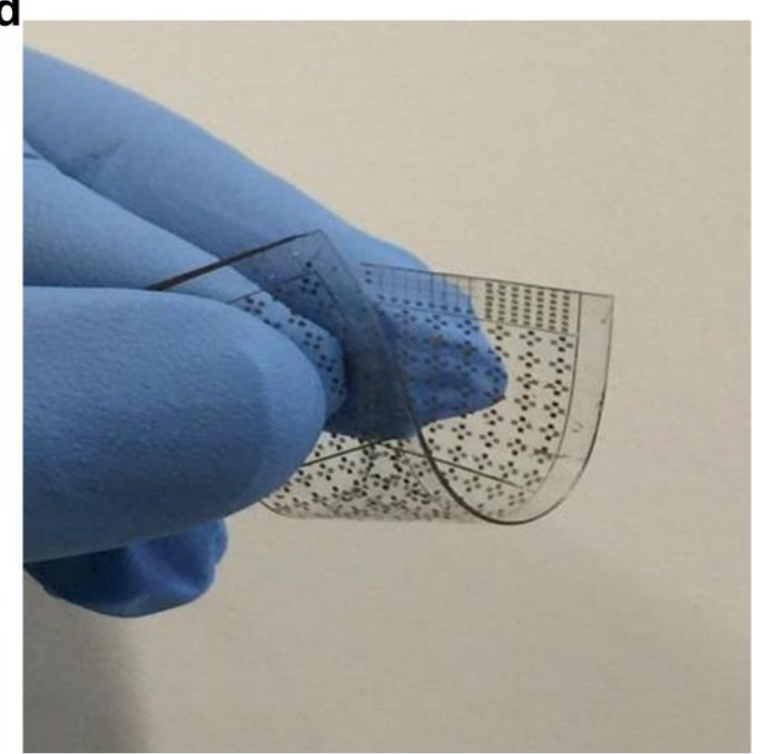

Fig. 1. 

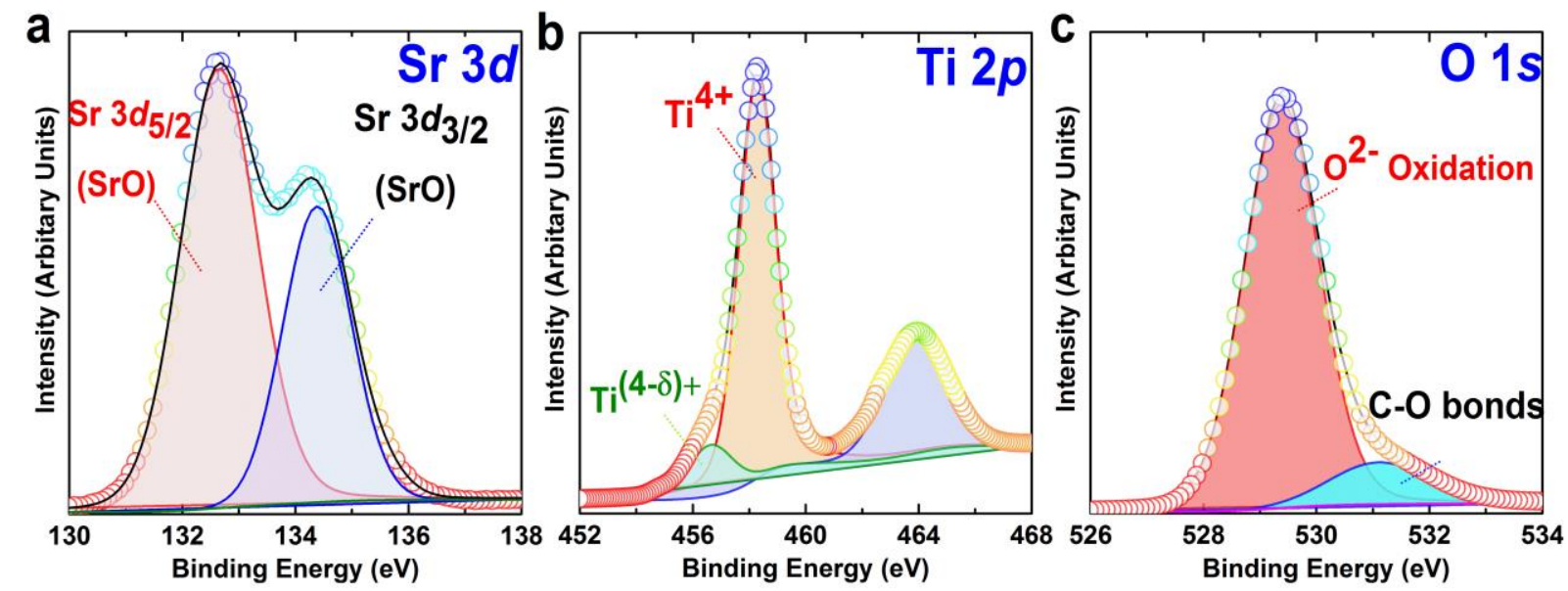

Fig. 2. 


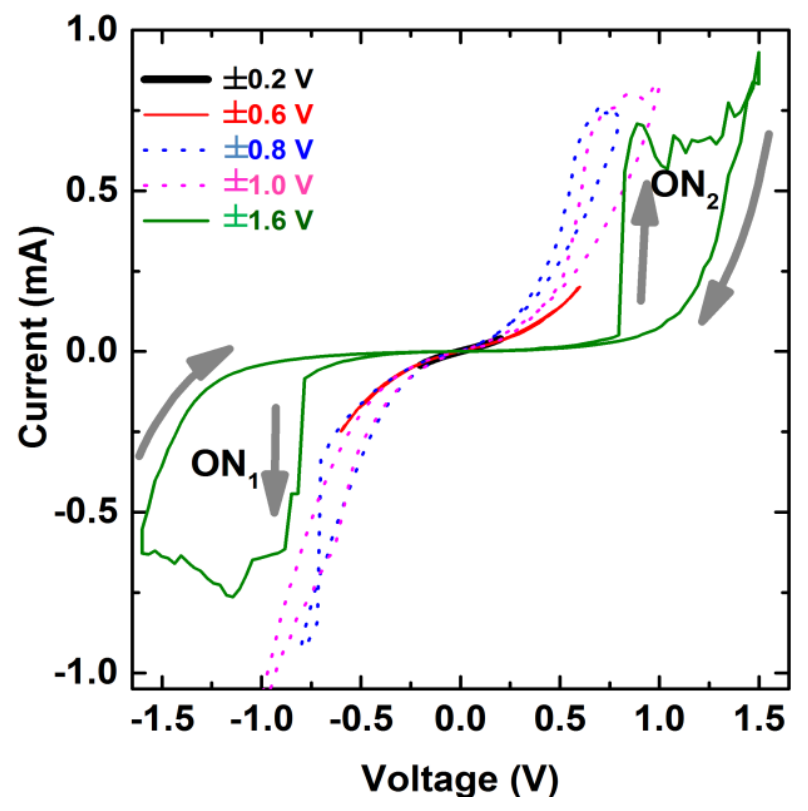

Fig. 3. 


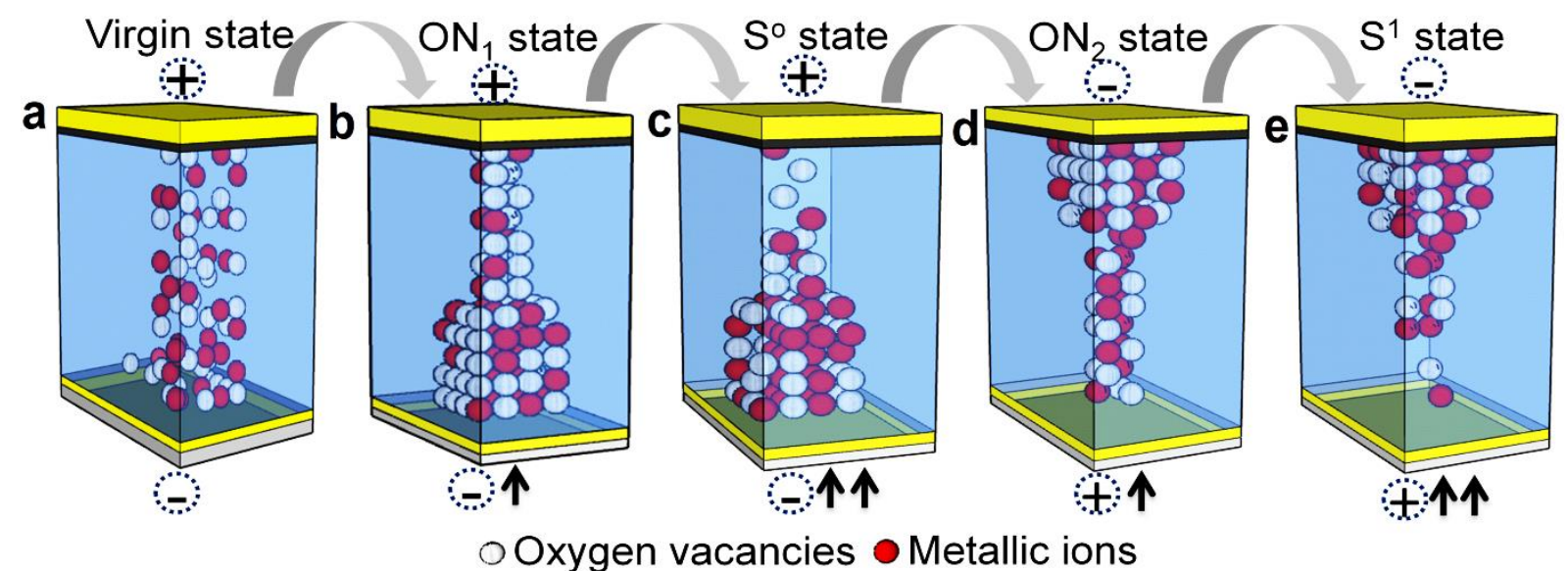

Fig. 4. 

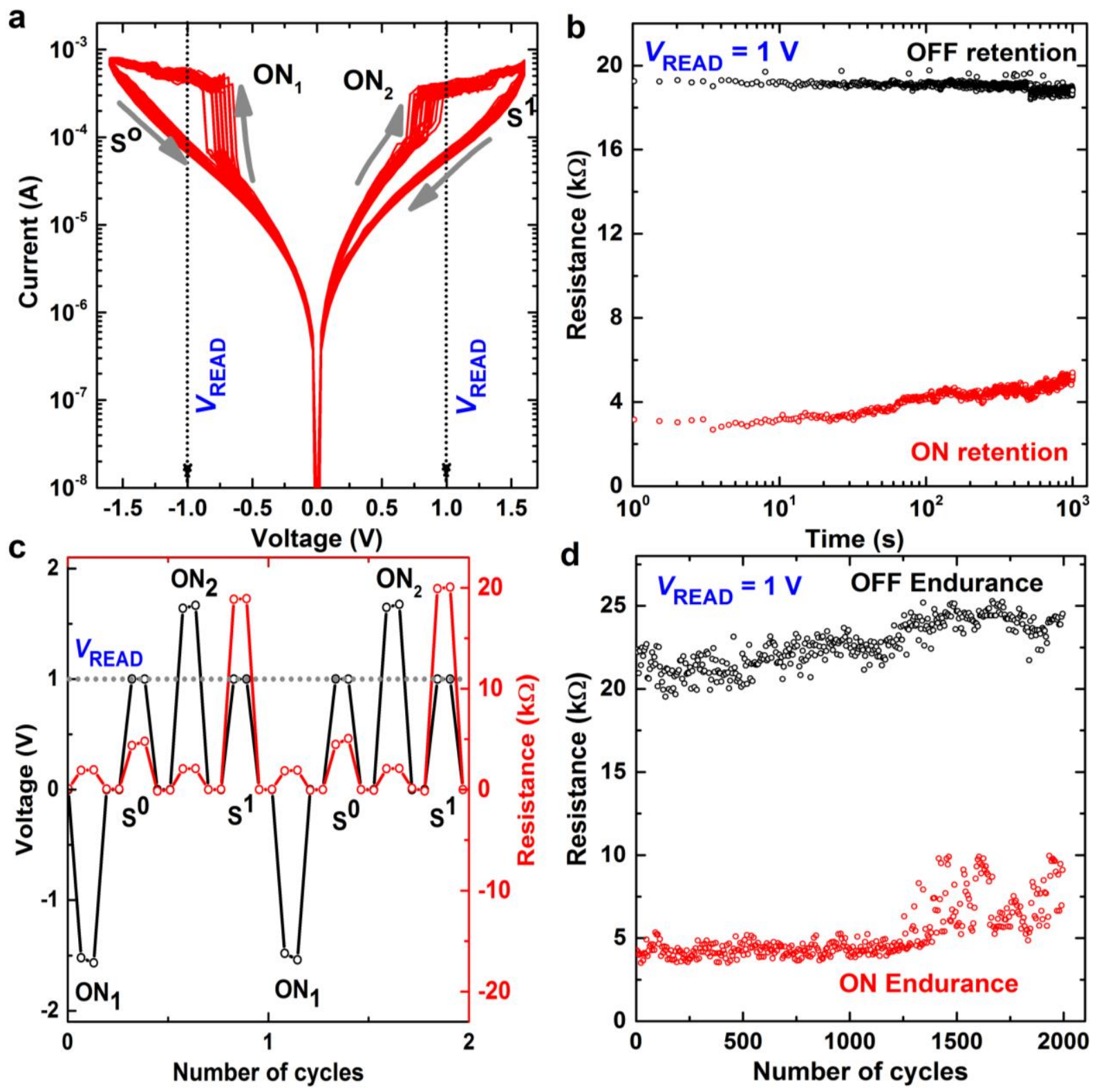

Fig. 5. 

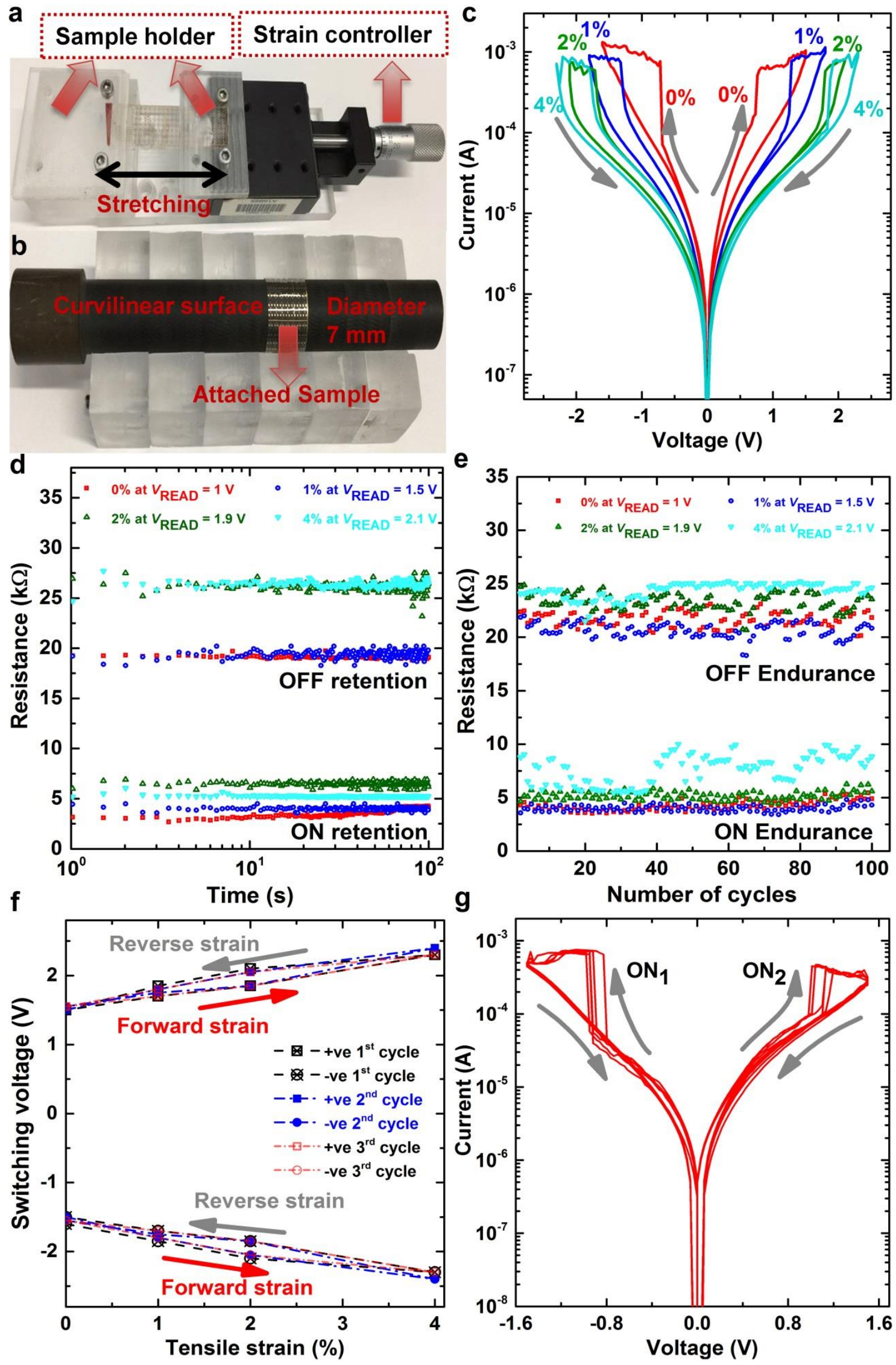

Fig. 6. 

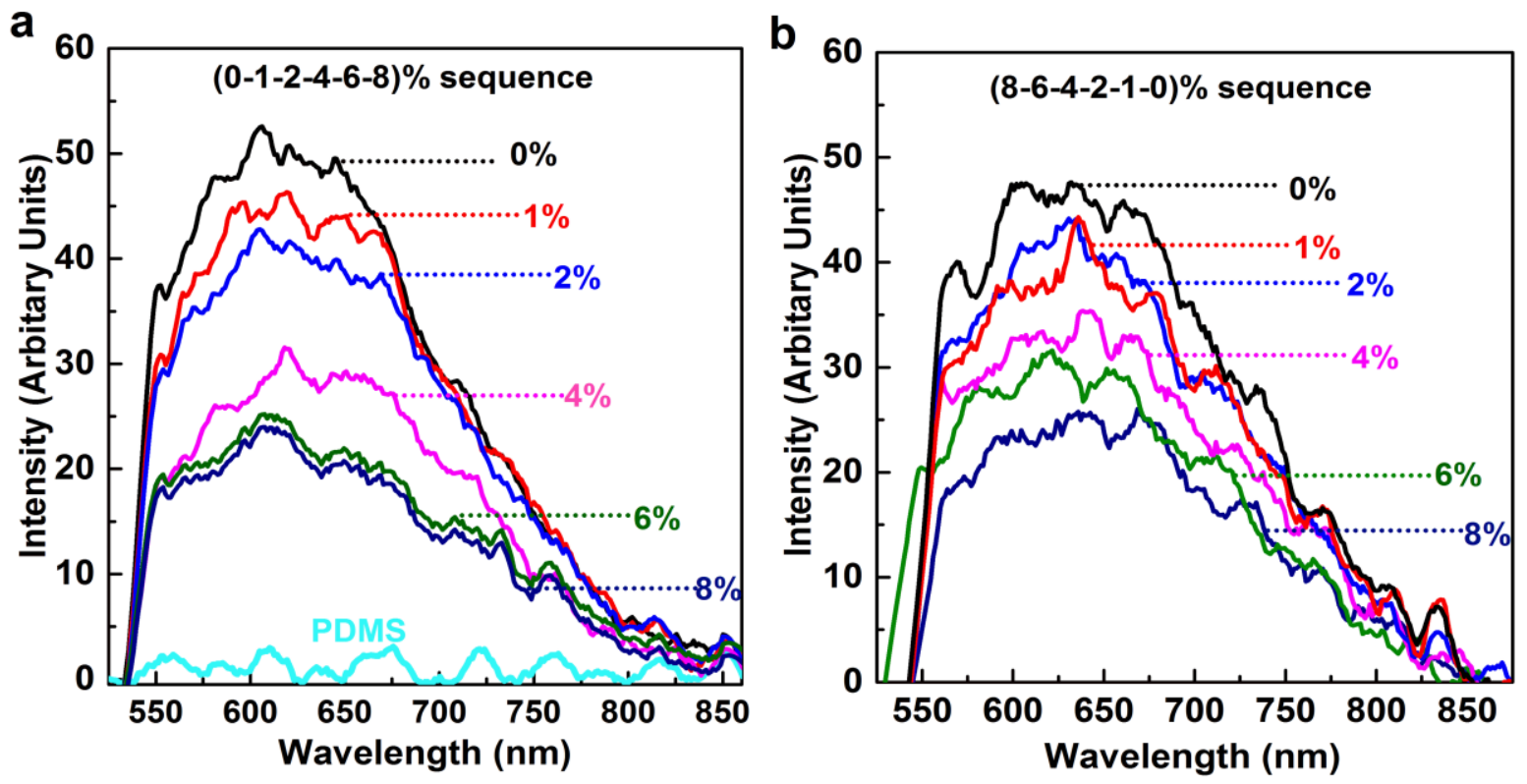

Fig. 7. 


\section{Supporting Information}

\section{Oxygen-deficient strontium titanate based stretchable resistive memories}

Md. Ataur Rahman*, Taimur Ahmed, Sumeet Walia, Sharath Sriram and Madhu Bhaskaran*

Functional Materials and Microsystems Research Group and the Micro Nano Research Facility, RMIT University, Melbourne, VIC 3001, Australia

*Corresponding authors email: s3576128@ student.rmit.edu.au madhu.bhaskaran@rmit.edu.au

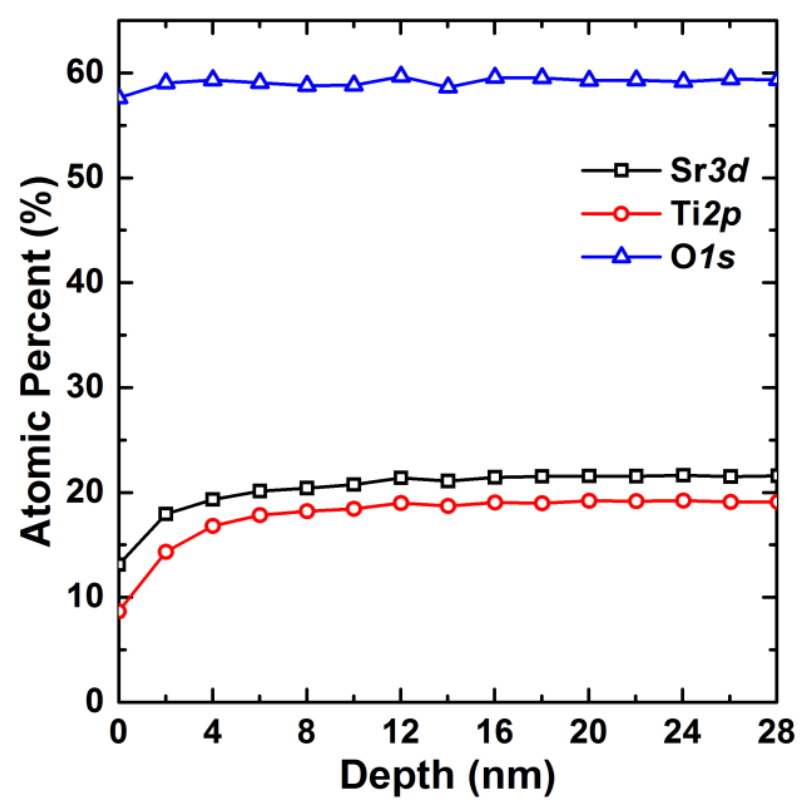

Figure S1. Atomic concentration of the major elements in room temperature. 

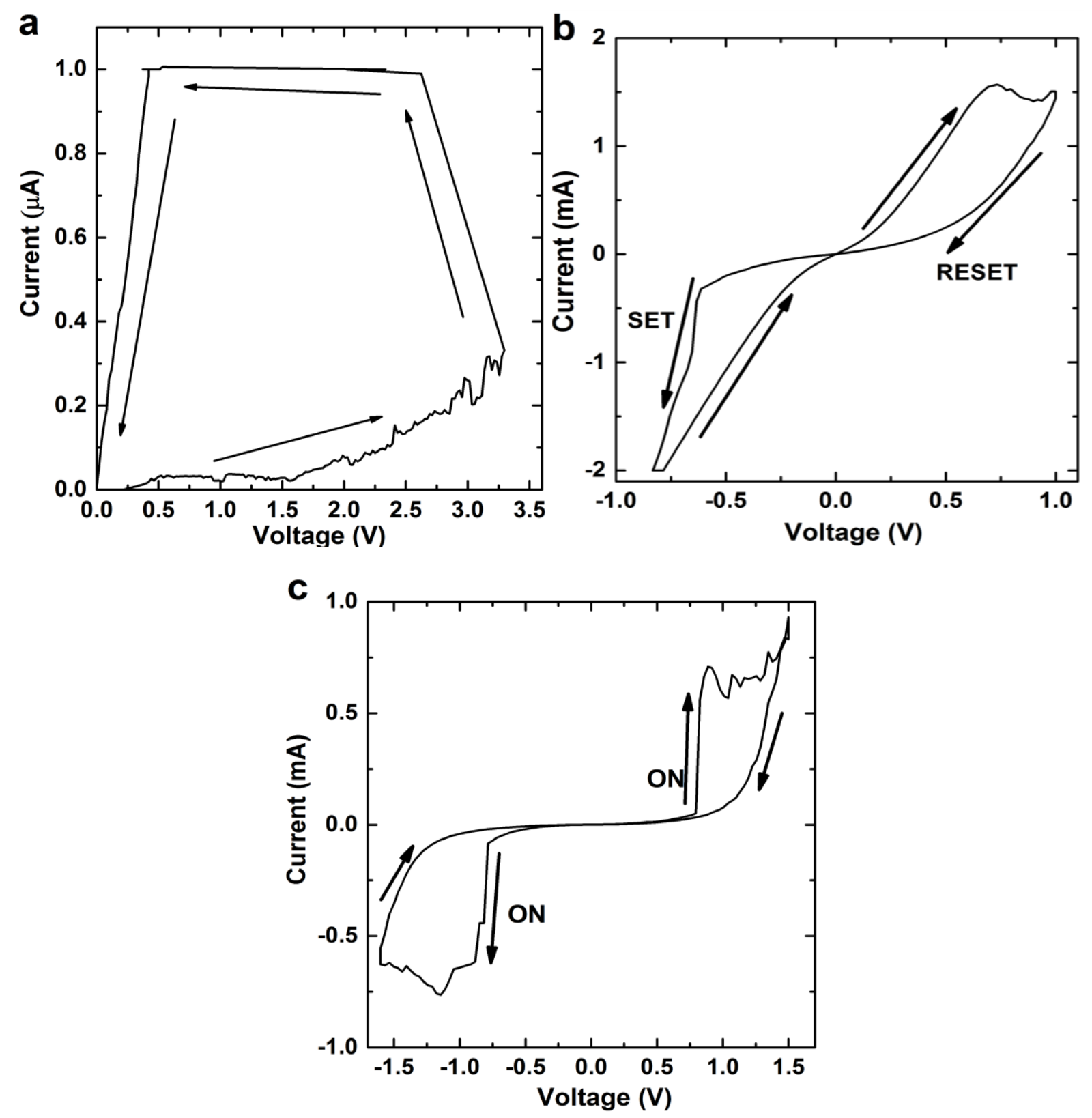

Figure S2. Switching behaviours comparison: (a) Electroforming process for $\mathrm{Pt} / \mathrm{Ti} / \mathrm{STO} / \mathrm{Pt} / \mathrm{Ti}$ on $\mathrm{SiO}_{2}$ substrate based devices. (b) $\mathrm{Pt} / \mathrm{Ti} / \mathrm{STO} / \mathrm{Pt} / \mathrm{Ti}$ on $\mathrm{SiO}_{2}$ substrate exhibits bipolar switching behaviour after electroforming process, and (c) Au/Ti/STO/Au/Cr on PDMS substrate shows complementary switching behaviour without electroforming process. 


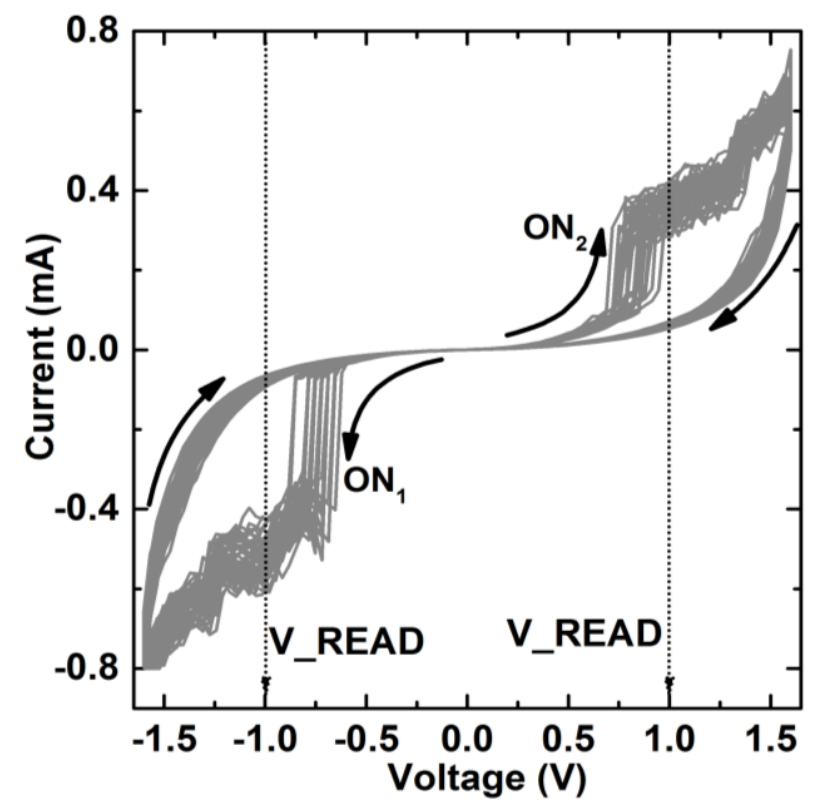

Figure S3. Threshold switching for 100 cycles in linear scale. 


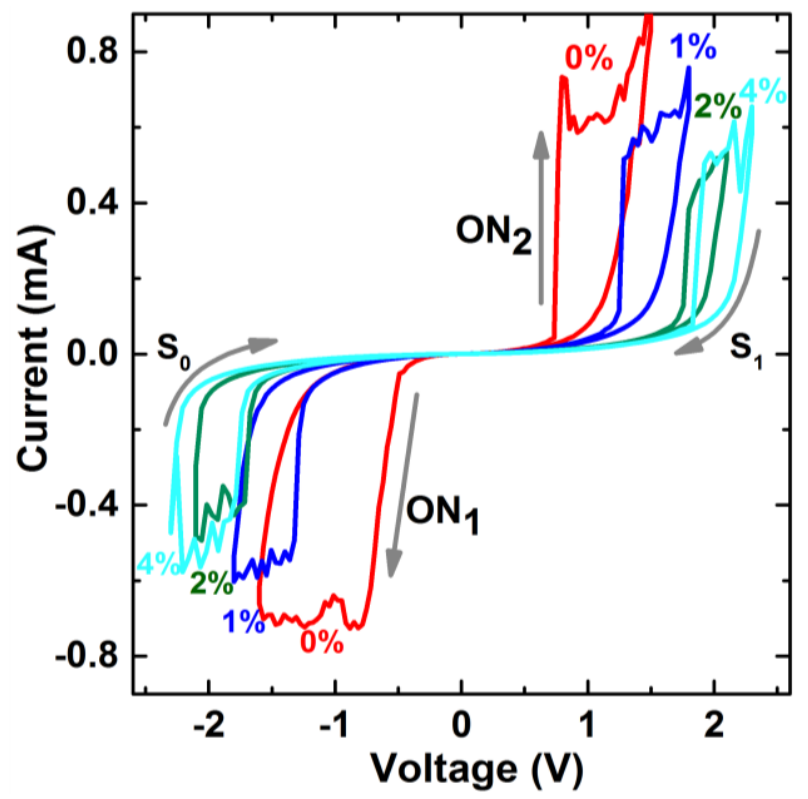

Figure S4. Switching under tensile strain from $0 \%$ to $4 \%$ for linear scale. 


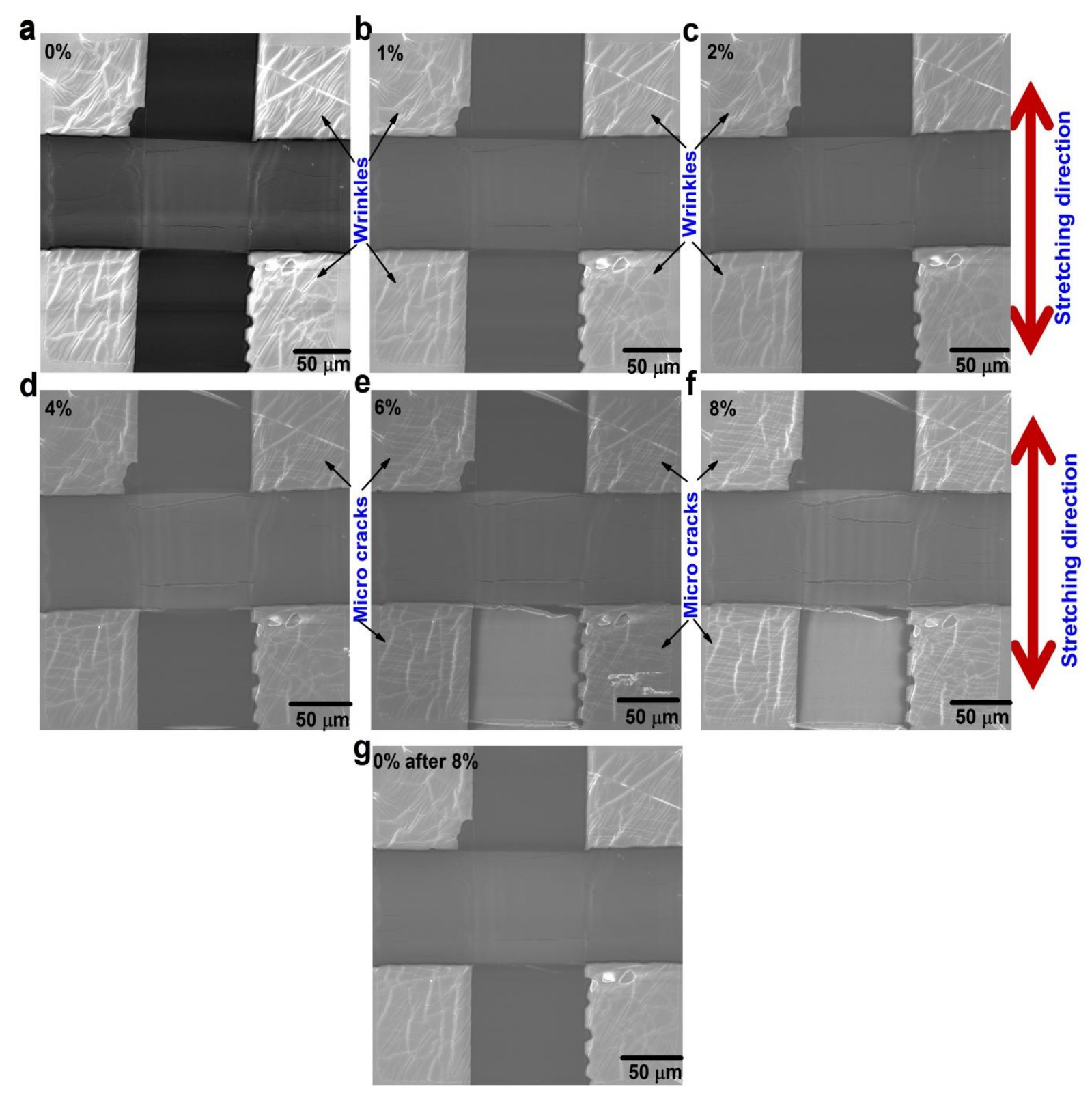

Figure S5. (a-f) Scanning electron micrographs of the electrodes and active layer under varying degree of strain from $0-8 \%$ and $(\mathrm{g})$ the morphology of the device after removal of strain. 


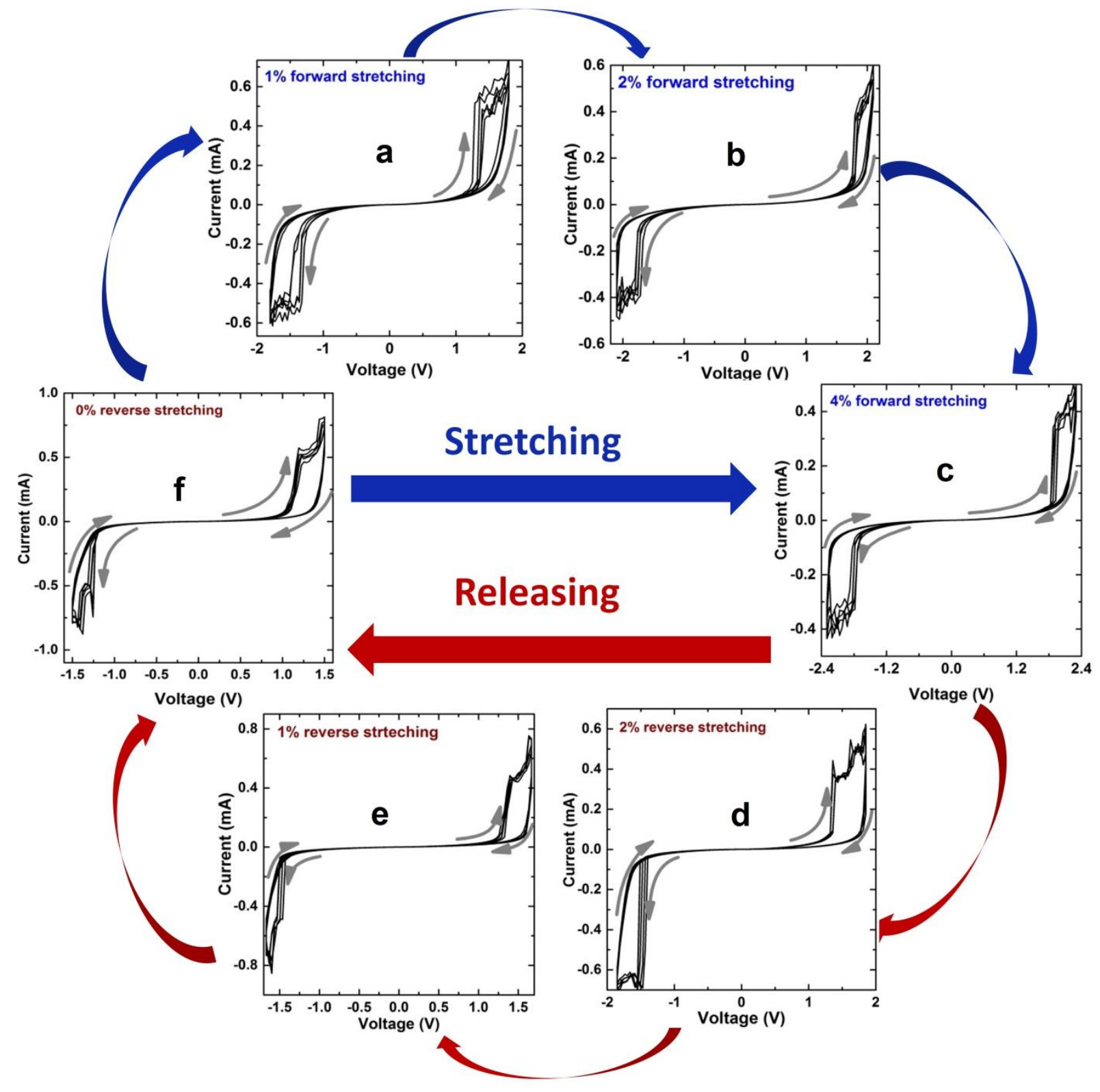

Figure S6. Stable switching behaviour during the stretching (a-c) and releasing (d-f). 


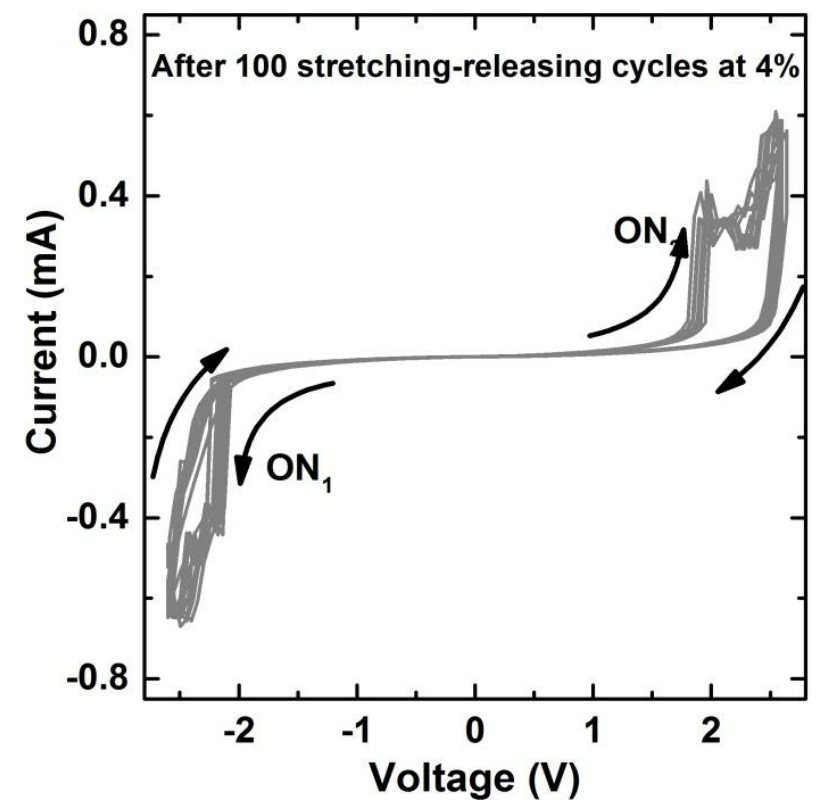

Figure S7. Threshold switching in linear scale after 100 stretch-release cycles at $4 \%$. 


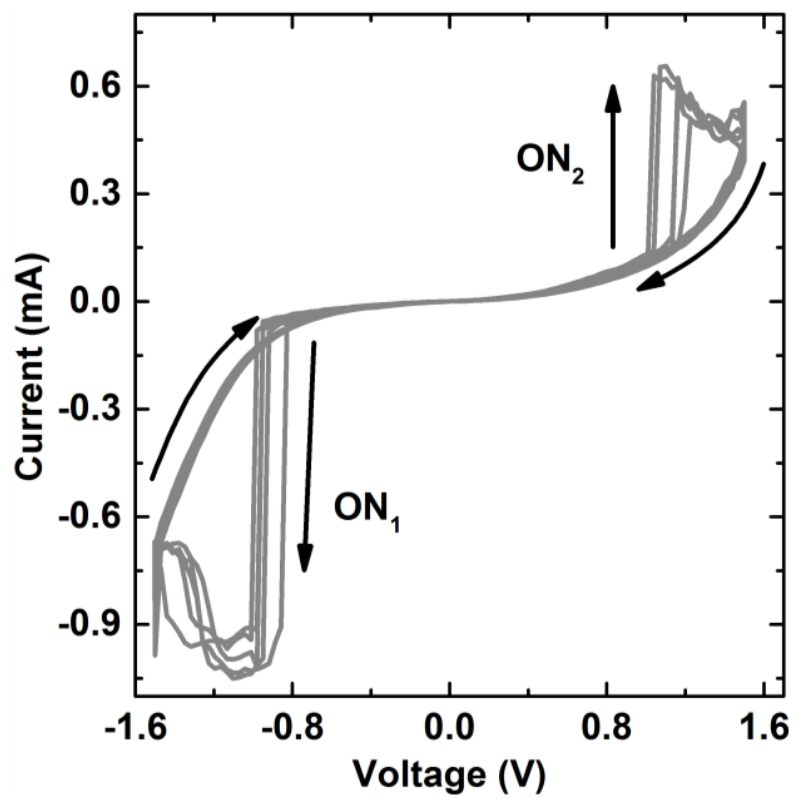

Figure S8. Switching on curvilinear surface with a radius of $3.5 \mathrm{~mm}$ in linear scale. 